$z-w$, take the limit as $w$ approaches $z$, and find $\mu^{\prime}(z)=$ $-\lambda(2 z) / \lambda^{4}(z)$, the correspondent of $\wp^{\prime}(z)=-\sigma(2 z) / \sigma^{4}(z)$.

If we wish to interpret this partial isomorphism in terms of Lucas' $U_{n}, V_{n}$, where $n$ is an integer, we may so do in the special case (cf. $\$ 1$ ) in which $q=1$. The derivatives $\lambda^{\prime}(z)$, $\mu^{\prime}(z), \cdots$ are then replaced by their equivalents in terms of $U(z), V(z)$ with $z$ finally replaced by $n$.

California Institute of Technology

\title{
AN INEQUALITY FOR DEFINITE HERMITIAN DETERMINANTS*
}

\author{
BY E. F. BECKENBACH
}

The proof of M. Ragnar Frisch's theorem, $\dagger$ The absolute value of a symmetric, definite determinant of real elements is at most equal to the product of the absolute values of the elements of the principal diagonal, may be generalized to establish the following theorem of which the above is clearly a special case: The absolute value of a definite Hermitian determinant is at most equal to the product of the absolute values of the elements of the principal diagonal.

An Hermitian determinant

$$
H \equiv\left|\begin{array}{c}
h_{11} \cdots h_{1 n} \\
\ldots \ldots . \\
h_{n 1} \cdots h_{n n}
\end{array}\right|
$$

is a determinant such that

$$
h_{r s}=\bar{h}_{s r}, \quad(r, s=1,2, \cdots, n),
$$

* Presented to the Society, February 23, 1929.

$\dagger$ Sur le théorème des déterminants de M. Hadamard, Comptes Rendus, vol. 185 (1927), p. 124. This is not a new theorem; see Bachmann, Die Arithmetik der Quadratischen Formen, 1923, pp. 250-251. It is more the method of proof than the result that makes Frisch's paper of interest. Though Bachmann does not give the generalized proof of the present paper, his proof holds equally for it. 
where $\bar{h}_{s r}$ designates the conjugate imaginary of $h_{s r}$. A definite Hermitian determinant is an Hermitian determinant such that the corresponding Hermitian form

$$
h \equiv \sum_{r, s=1}^{n} h_{r s} x_{r} \bar{x}_{s}, \quad\left(h_{r s}=\bar{h}_{s r}\right)
$$

is definite. The reader may readily verify the fact that an Hermitian form can never take on a complex value. An Hermitian form which is positive (negative) or zero for all values of the variables is said to be positive (negative) definite; in either case, the form is said to be definite.

We can suppose the definite form (2) corresponding to the definite determinant (1) to be positive definite, for otherwise we could consider the form

$$
-h \equiv \sum_{r, s=1}^{n}\left(-h_{r s}\right) x_{r} \bar{x}_{s}, \quad\left(h_{r s}=\bar{h}_{s r}\right),
$$

which would be positive definite, and whose corresponding determinant would be equal in absolute value to $H$. And we can suppose the form to be non-singular, for if the determinant were equal to zero the theorem would be trivial.

The determinant

$$
F(\lambda) \equiv\left|\begin{array}{cccc}
h_{11}-\lambda & h_{12} & \cdots & h_{1 n} \\
h_{21} & h_{22}-\lambda & \cdots & h_{2 n} \\
\cdots \cdots & \ldots & \cdots & \cdots \\
h_{n 1} & h_{n 2} & \cdots & h_{n n}-\lambda
\end{array}\right|
$$

may be expanded into a polynomial in $\lambda$ of degree $n$, where the constant term, $H$, is unequal to zero. Hence we can construct*

$$
F(\lambda) \equiv \frac{[P(\lambda)]^{2}-\lambda}{Q(\lambda)},
$$

where $P(\lambda)^{-}$and ${ }^{-} Q(\lambda)^{-}$are polynomials in $\lambda$ and $P(\lambda)$ is of

* See Bôcher, Introduction to Higher Algebra, pp. 297-299. 
degree less than $n$. As is well known, ${ }^{*}$ the roots of the characteristic equation,

$$
F(\lambda)=0,
$$

are all real and greater than zero. Note, then, in the above construction, that the coefficients in $P(\lambda)$ are all real.

Let

$$
M \equiv\left(\begin{array}{c}
h_{11} \cdots h_{1 n} \\
\cdots \cdots \\
h_{n 1} \cdots h_{n n}
\end{array}\right)
$$

be the matrix corresponding to $H$. By a fundamental theorem, $\uparrow$

Hence, (3) gives

$$
F(M) \equiv 0
$$

$$
[P(M)]^{2} \equiv M
$$

It is easy to show that $P(M)$, being a polynomial with real coefficients in an Hermitian matrix, is itself an Hermitian matrix of the same order. Now let

Then from (4),

$$
P(M) \equiv\left(\begin{array}{c}
b_{11} \cdots b_{1 n} \\
\cdots \ldots \\
b_{n 1} \cdots
\end{array}\right), \quad\left(b_{n n}=b_{n r}\right)
$$

$$
h_{i i}=\sum_{j=1}^{n} b_{i j} \bar{b}_{i j}, \quad(i=1,2, \cdots, n) .
$$

Whether the form is positive or negative definite, we still have

$$
\bmod h_{i i}=\sum_{j=1}^{n} b_{i j} \bar{b}_{i j}, \quad(i=1,2, \cdots, n),
$$

where by $\bmod h_{i i}$ we mean the modulus, or absolute value, of $h_{i i}$.

* See Kowalewski, Einführung in die Determinantentheorie, Leipzig, 1909, pp. 280-284.

† See Bôcher, loc. cit., p. 296. 
I am indebted to Professor H. E. Bray for the following proof of Hadamard's Theorem. Given the matrix of real or complex elements

$$
\alpha \equiv\left(\begin{array}{c}
\alpha_{1} \\
\alpha_{2} \\
\vdots \\
\alpha_{n}
\end{array}\right), \quad \alpha_{i} \equiv\left(\alpha_{i 1}, \alpha_{i 2}, \cdots, \alpha_{i n}\right),
$$

consider the matrix

where

$$
\beta \equiv\left(\begin{array}{c}
\beta_{1} \\
\beta_{2} \\
\vdots \\
\dot{\beta}_{n}
\end{array}\right)
$$

$$
\begin{aligned}
& \beta_{1}=\alpha_{1}, \\
& \cdots \cdots \\
& \beta_{i}=\sum_{j=1}^{i-1} k_{i}^{i} \beta_{i}+\alpha_{i} .
\end{aligned}
$$

We note that

$$
|\beta|=|\alpha| \text {. }
$$

Choose the $k_{l}^{i}$ so that, for given $i$ and all $j<i$,

$$
\left(\beta_{i}, \bar{\beta}_{j}\right) \equiv \beta_{i 1} \bar{\beta}_{i 1}+\beta_{i 2} \bar{\beta}_{j 2}+\cdots+\beta_{i n} \bar{\beta}_{j n}=0 .
$$

That is,

$$
\left(\left[\sum_{l=1}^{i-1} k_{l}^{i} \beta_{l}+\alpha_{i}\right], \bar{\beta}_{j}\right)=k_{j}^{i}\left(\beta_{j}, \bar{\beta}_{j}\right)+\left(\alpha_{i}, \bar{\beta}_{j}\right)=0,
$$

or

Hence

$$
k_{j}^{i}=-\frac{\left(\alpha_{i}, \bar{\beta}_{j}\right)}{\left(\beta_{j}, \bar{\beta}_{j}\right)} .
$$

$$
\sum_{j=1}^{i-1} \bar{k}_{i}^{i}\left(\alpha_{i}, \bar{\beta}_{j}\right)=-\sum_{j=1}^{i-1} k_{j}{ }^{i} \bar{k}_{j}{ }^{i}\left(\beta_{i}, \bar{\beta}_{j}\right),
$$

which is real and negative. Now

$$
0 \leqq\left(\beta_{i}, \bar{\beta}_{i}\right)=\left(\left[\sum_{j=1}^{i-1} k_{j}^{i} \beta_{j}+\alpha_{i}\right],\left[\sum_{j=1}^{i-1} \bar{k}_{j}^{i} \bar{\beta}_{i}+\bar{\alpha}_{i}\right]\right),
$$


whence

But

$$
\begin{aligned}
& 0 \leqq\left(\beta_{i} \bar{\beta}_{i}\right)= \sum_{j=1}^{i-1} k_{j}{ }^{i} \bar{k}_{j}{ }^{i}\left(\beta_{j}, \bar{\beta}_{j}\right) \\
&+\sum_{j=1}^{i-1} \bar{k}_{j}{ }^{i}\left(\alpha_{i}, \bar{\beta}_{j}\right) \\
&+\sum_{j=1}^{i-1} k_{j}{ }^{i}\left(\bar{\alpha}_{i}, \beta_{j}\right)+\left(\alpha_{i}, \bar{\alpha}_{i}\right) \\
&=\left(\alpha_{i}, \bar{\alpha}_{i}\right)-\sum_{j=1}^{i-1} k_{j}{ }^{i} \bar{k}_{j}{ }^{i}\left(\beta_{j}, \bar{\beta}_{j}\right) \leqq\left(\alpha_{i}, \bar{\alpha}_{i}\right)
\end{aligned}
$$

Hence

$$
|\alpha| \cdot|\bar{\alpha}|=|\beta| \cdot|\bar{\beta}|=\prod_{i=1}^{n}\left(\beta_{i}, \bar{\beta}_{i}\right)
$$

or

$$
|\alpha| \cdot|\bar{\alpha}| \leqq \prod_{i=1}^{n}\left(\alpha_{i}, \bar{\alpha}_{i}\right)
$$

$$
\bmod |\alpha| \leqq \prod_{i=1}^{n}\left[\left(\alpha_{i}, \bar{\alpha}_{i}\right)\right]^{1 / 2}
$$

This inequality expresses Hadamard's Theorem: The $a b$ solute value of any determinant is at most equal to the product of the norms (either horizontal or vertical) of the determinant. The quantities

$$
+\left[\left(\alpha_{i}, \bar{\alpha}_{i}\right)\right]^{1 / 2}, \quad \text { or }+\left(\sum_{j=1}^{n} \alpha_{i j} \bar{\alpha}_{i j}\right)^{1 / 2}, \quad(i=1,2, \cdots, n),
$$

are called the horizontal norms of the determinant $|\alpha|$, and a similat definition holds for the vertical norms.

Applying this theorem to the determinant $|P(M)|$, we get

$$
\bmod |P(M)|^{2} \leqq \prod_{i=1}^{n}\left(\sum_{j=1}^{n} b_{i j} \bar{b}_{i j}\right)
$$

Equations (4), (5), and (6) give

$$
\bmod H \leqq \prod_{i=1}^{n}\left(\bmod h_{i i}\right) . \quad \text { Q.E.D. }
$$

The Rice Institute 